JOURNAL OF

SYMPLECTIC GEOMETRY

Volume 9, Number 1, 1-9, 2011

\title{
ON THE ALGEBRAIC INDEPENDENCE OF HAMILTONIAN CHARACTERISTIC CLASSES
}

\author{
Światoseaw Gal, Jarek Kędra, and Aleksy Tralle
}

We prove that Hamiltonian characteristic classes defined as fibre integrals of powers of the coupling class are algebraically independent for generic coadjoint orbits.

\section{Introduction}

Let $(M, \omega)$ be a closed symplectic manifold of dimension $2 n$ and let

$$
(M, \omega) \stackrel{i}{\rightarrow} E \stackrel{\pi}{\rightarrow} B
$$

be a Hamiltonian fibration over a simply connected base. It means that the structure group of the fibration is contained in the $\operatorname{group} \operatorname{Ham}(M, \omega)$ of Hamiltonian diffeomorphisms of $(M, \omega)$. There exists a cohomology class $\Omega \in H^{2}(E)$ that is uniquely defined by the following two conditions:

$$
\begin{aligned}
i^{*} \Omega & =[\omega], \\
\pi_{!}\left(\Omega^{n+1}\right) & =0 .
\end{aligned}
$$

It is called the coupling class. The existence of the coupling class and its basic properties are discussed in $[\mathbf{1}, \mathbf{5}]$.

Since the fibre integration is functorial the coupling class is natural in the sense that the coupling class of the pull-back bundle is the pull-back of the coupling class. One defines the following characteristic classes of Hamiltonian fibration:

$$
\mu_{k}(E):=\pi_{!}\left(\Omega^{n+k}\right) \in H^{2 k}(B) .
$$

The fundamental question arises if these classes are nontrivial and to what extent are they algebraically independent in the cohomology ring $H^{*}(B \operatorname{Ham}(M, \omega))$ of the classifying space of the group of Hamiltonian diffeomorphisms.

The first result about the algebraic independence was proved by Reznikov for $\mathbb{C P}^{n}\left(\left[\mathbf{1 0}\right.\right.$, Section 1.3]) and it states that the classes $\mu_{k}$ are algebraically 
independent for $k=2, \ldots, n+1$. Reznikov also suggested that this result may be true for any coadjoint orbit of a compact Lie group. The main result of the present note states that Reznikov's claim is correct generically.

Theorem 1.1. Let $G$ be a compact semisimple Lie group and let

$$
\mathcal{K}:=\left\{k \in \mathbb{N} \mid \pi_{2 k}(B G) \otimes \mathbb{Q} \neq 0\right\} .
$$

There exists a nonempty Zariski open subset $A \subset \mathfrak{g}^{\vee}$ in the dual of the Lie algebra of $G$ such that for any $\xi \in A$ the coadjoint orbit $M_{\xi}$ of $\xi$ satisfies the following. The classes $\mu_{k} \in H^{2 k}\left(B \operatorname{Ham}\left(M_{\xi}\right)\right)$ are algebraically independent for $k \in \mathcal{K}$.

The proof of this theorem amounts to the calculation of the characteristic classes $\mu_{k}$ for the universal fibration $M_{\xi} \rightarrow B G_{\xi} \rightarrow B G$. Then we use the fact that the cohomology ring $H^{*}(B G)$ is a polynomial ring generated by elements with degrees in $\mathcal{K}$. This follows from results about cohomology of classifying spaces (see [7]) and basic rational homotopy theory.

Corollary 1.1. Let $G$ be a compact simple Lie group different from $\mathrm{SO}(4 k)$ There exists a nonempty Zariski open subset $A \subset \mathfrak{g}^{\vee}$ in the dual of the Lie algebra of $G$ such that for any $\xi \in A$ the coadjoint orbit $M_{\xi}$ of $\xi$ satisfies the following. The homomorphism $H^{*}\left(B \operatorname{Ham}\left(M_{\xi}\right)\right) \rightarrow H^{*}(B G)$ induced by the action is surjective and its image is generated by the classes $\mu_{k}$.

Remark 1.1. We exclude the orthogonal group $\mathrm{SO}(4 k)$ because there are two generators in $H^{4 k}(B \mathrm{SO}(4 k))$, the Euler class and the $k$ th Pontryagin class [7, Theorem 3.19]. We shall show in Section 3.4 that the Euler class is a nonzero multiple of the class $\mu_{2 k}$ for a bundle over $S^{4 k}$ with fibre $\mathrm{SO}(4 k) / U(2 k)$.

Remark 1.2. In order to prove the surjectivity of the homomorphism $H^{*}(B \operatorname{Ham}(M, \omega)) \rightarrow H^{*}(B G)$ one can use characteristic classes defined by integrating products of the equivariant Chern classes [2] or use different arguments [3].

In Section 3, we provide various examples of coadjoint orbits for which the algebraic independence does or does not hold. The simplest manifolds with the class $\mu_{3}$ trivial in $H^{*}(B G)$ are the complex grassmannian $G(2,4)$ of planes in $\mathbb{C}^{4}$ and the flag manifold $\mathrm{SU}(3) / T$ for a certain invariant symplectic form.

1.1. Conventions. Throughout the paper $H^{*}(X)$ denotes the cohomology of $X$ with real coefficients.

When we say that a statement holds for a generic element $x \in V$ of an algebraic variety we mean that there exists a Zariski open subset $S \subset V$ such that for every element $x \in S$ the statement holds. 


\section{Proof of the main result}

2.1. Flag manifolds. If a cohomology class evaluates nontrivially on a sphere then it is not the sum of the products of classes of lower degree. This simple observation allows one to prove the algebraic independence of the characteristic classes for flag manifolds.

Lemma 2.1. Let $G$ be a compact and connected semisimple group. Let $\omega$ be a generic homogeneous symplectic form on the flag manifold $G / T$. Then for a rationally nontrivial homotopy class $f: S^{2 k} \rightarrow B G$ the induced Hamiltonian bundle has a nontrivial class $\mu_{k}$.

Proof. Consider the following pull-back diagram

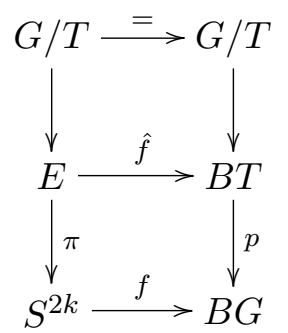

Let $\sigma \in H^{2 k}\left(S^{2 k}\right)$ be a generator and let $\sigma=f^{*}(\alpha)$ for some $\alpha \in H^{2 k}(B G)$. Notice that this implies that $k>1$. Since the cohomology of $B T$ is generated by classes of degree two the pull back

$$
\pi^{*}(\sigma)=\pi^{*}\left(f^{*}(\alpha)\right)=\hat{f}^{*}\left(p^{*}(\alpha)\right)
$$

is a sum of products of classes of degree two. This implies that the cohomology ring $H^{*}(E)$ is generated by degree two classes. Moreover, the inclusion of the fibre induces an isomorphism $H^{2}(E) \cong H^{2}(G / T)$.

Let $\operatorname{dim} G / T=2 n$ and hence the dimension of the total space $E$ is equal to $2(n+k)$. We claim that there exists a Zariski open subset of $H^{2}(E)$ consisting of classes whose $(n+k)$ th power is nonzero. Since taking the highest power defines an algebraic map $H^{2}(E) \rightarrow H^{2(n+k)}(E)=\mathbb{R}$ it is enough to find just one class with nontrivial highest power. Observe that the symmetric map

$$
H^{2}(E)^{\otimes(n+k)} \ni a_{1} \otimes \cdots \otimes a_{n+k} \mapsto a_{1} \cdot \ldots \cdot a_{k+n} \in H^{n+k}(E)
$$

is nontrivial as $H^{*}(E)$ is generated in dimension 2 and $E$ is closed and oriented. Since any multilinear symmetric map is determined (via the polarisation formula) by a polynomial map, the map

$$
H^{2}(E) \ni a \mapsto a^{n+k} \in H^{n+k}(E)
$$

is nontrivial. 
Let $\omega \in \Omega^{2}(G / T)$ be a generic $G$-invariant symplectic form. Then the associated coupling class $\Omega \in H^{2}(E)$ is such that $\Omega^{n+k} \neq 0$ and hence

$$
\left\langle\mu_{k}(E),\left[S^{2 k}\right]\right\rangle=\left\langle\pi_{!}\left(\Omega^{n+k}\right),\left[S^{2 k}\right]\right\rangle=\left\langle\Omega^{n+k},[E]\right\rangle \neq 0 .
$$

Corollary 2.1. Let $\mathcal{K}=\left\{k \in \mathbb{N} \mid \pi_{2 k}(B G) \otimes \mathbb{Q} \neq 0\right\}$. For a generic homogeneous symplectic form on a flag manifold $G / T$ the classes $\mu_{k} \in$ $H^{2 k}(\operatorname{BHam}(G / T))$ are algebraically independent for $k \in \mathcal{K}$. Moreover, these classes cannot be generated by classes of smaller degrees.

Proof. Let $\xi \in \mathfrak{g}^{\vee}$ be an element such that its coadjoint orbit $M_{\xi}$ is diffeomorphic to a flag manifold $G / T$. It follows from Lemma 2.1 that for each $k \in \mathcal{K}$ the class $\mu_{k}(E) \in H^{*}\left(S^{2 k}\right)$ is nonzero for $\xi$ in an open and dense subset of $\mathfrak{g}^{\vee}$. Taking the intersection of these subsets for all $k \in \mathcal{K}$ we obtain an open and dense subset for which the classes are nontrivial for Hamiltonian fibrations over spheres. Since these classes do not vanish on spheres they cannot be generated by classes of smaller degrees. The algebraic independence follows from the fact that $H^{*}(B G)$ is a free polynomial algebra.

2.2. The general case. The cohomology ring $H^{*}(B G)$ is isomorphic to the ring of the invariants $S\left(\mathfrak{g}^{\vee}\right)^{G}$ of the Lie algebra of $G$ and to the ring of polynomials $S\left(\mathfrak{t}^{\vee}\right)^{W}$ on the Lie algebra of the maximal torus $T \subset G$ invariant under the action of the Weyl group. Let $M_{\xi}$ be the coadjoint orbit of $\xi \in \mathfrak{g}^{\vee}$. Every class $\mu_{k} \in H^{2 k}(B G)$ for the orbit $M_{\xi}$ defines an invariant polynomial $p_{\xi, k} \in S\left(\mathfrak{g}^{\vee}\right)^{G}$ as follows.

Let $X \in \mathfrak{g}$ be an element generating a circle action and let

$$
c_{X}: B S^{1}=\mathbb{C P}^{\infty} \rightarrow B G
$$

be the corresponding classifying map. We define

$$
p_{\xi, k}(X):=\left\langle c_{X}{ }^{*}\left(\mu_{k}\right), \mathbb{C P}^{k}\right\rangle \in \mathbb{R}
$$

and extend $p_{\xi, k}$ to the whole of $\mathfrak{g}$ by continuity. Notice that a function $H: M_{\xi} \rightarrow \mathbb{R}$ given by

$$
H\left(\operatorname{Ad}_{g}^{\vee}(\xi)\right)=\left\langle X, \operatorname{Ad}_{g}^{\vee}(\xi)\right\rangle
$$

is the Hamiltonian function for the action generated by $X \in \mathfrak{g}$. The next lemma follows from [3, Lemma 3.6] and it shows that $p_{\xi, k}$ is indeed an invariant polynomial.

Lemma 2.2. Let $X \in \mathfrak{g}$. The following formula holds true:

$$
p_{\xi, k}(X)=(-1)^{k}\left(\begin{array}{c}
n+k \\
k
\end{array}\right) \cdot \int_{G}\left\langle X, \operatorname{Ad}_{g}^{\vee}(\xi)\right\rangle^{k} \operatorname{vol}_{G} .
$$


Corollary 2.2. Let $(M, \omega)$ be a nontrivial coadjoint orbit of a semisimple Lie group $G$. Then the class $\mu_{2 k} \in H^{4 k}(B \operatorname{Ham}(M, \omega))$ is nontrivial for every positive integer $k \in \mathbb{N}$.

2.3. Proof of Theorem 1.1. It follows from Corollary 2.1 that the relevant classes $\mu_{k}$ are algebraically independent in $H^{*}(B G)$ for a generic $\xi$ defining a flag manifold. Since the algebraic independence is an open condition it follows that the polynomials $p_{\xi, k}$ are algebraically independent for a generic $\xi \in \mathfrak{g}^{\vee}$ and $k \in \mathcal{K}$.

Remark 2.1. Observe that $p_{\xi, k}(X)$ defines a bi-invariant polynomial of degree $k$ on the tensor product $\mathfrak{g} \otimes \mathfrak{g}^{\vee}$. The algebra of $G$-bi-invariant polynomials on $\mathfrak{g} \otimes \mathfrak{g}^{\vee}$ is isomorphic to the algebra of polynomials on $\mathfrak{t} \otimes \mathfrak{t}^{\vee}$ bi-invariant for the Weyl group of $G$.

There are two interesting Zariski open subsets of the dual Lie algebra $\mathfrak{t}^{\vee}$ of the maximal torus $T \subset G$. One consists of those elements giving flag manifolds as coadjoint orbits. And this is just the union of the interiors of the Weyl chambers. The other consists of elements such that the characteristic classes associated with their coadjoint orbits are algebraically independent. In the next section we discuss examples showing that these two sets do not contain each other. In particular, the algebraic independence holds not only for flag manifolds.

\section{Examples}

3.1. Coadjoint orbits of $\mathbf{S U}(\boldsymbol{n})$. As we mentioned in the Introduction, Reznikov proved that the classes $\mu_{k}$ are algebraically independent in $H^{*}\left(B \operatorname{Ham}\left(\mathbb{C P}^{n-1}\right)\right)$ for $k=2, \ldots, n$ (see [3, Proposition 1.7] for an alternative proof). Hence it follows that these classes are also algebraically independent for any coadjoint orbit of $\mathrm{SU}(n)$ which is close to $\mathbb{C} \mathbb{P}^{n-1}$.

More precisely, since $\mathrm{SU}(n)$ is simple the coadjoint representation is isomorphic (via the Killing form) to the adjoint representation. After this identification the complex projective space $\mathbb{C P}^{n-1}$ is the adjoint orbit of the diagonal matrix

$$
\xi=\operatorname{diag}[-i,-i, \ldots,-i,(n-1) i] \in \mathfrak{s u}(n) .
$$

By an adjoint orbit close to $\mathbb{C P}^{n-1}$ we mean the orbit of an element $\xi^{\prime}$ from a suitably small neighbourhood of $\xi$.

3.2. The failure of the algebraic independence. Checking directly whether a class $\mu_{k}$ is a polynomial in lower degree classes seems to be complicated in general. However, one can make an interesting claim if $\operatorname{dim} H^{2 k}(B G)=1$. The latter condition is always true for a simple compact Lie group $G$ and $k=2$, and for a single odd number $k$ if $G$ has one of 
the groups $\mathrm{SU}(n), \mathrm{SO}(4 k+2)$ or $E_{6}$ as a factor. We shall discuss concrete examples in the next sections.

Proposition 3.1. Let $G$ be a compact Lie group and let $m \in \mathbb{N}$ be a number for which $\pi_{2 m}(B G) \otimes \mathbb{Q}=H^{2 m}(B G ; \mathbb{Q})=\mathbb{Q}$. Let $u \in S\left(\mathfrak{g}^{\vee}\right)^{G}$ be a nontrivial invariant polynomial of degree $m$. The class $\mu_{m} \in H^{2 m}(B G)$ is trivial for the coadjoint orbit $M_{\xi}$ if and only if $u(\xi)=0$.

Proof. It follows from the hypothesis and the isomorphism $H^{*}(B G)=$ $S\left(\mathfrak{g}^{\vee}\right)^{G}$ that the polynomial $u$ is unique up to a constant. Since the polynomial $p_{\xi, m}(X)$ is bi-invariant on the tensor product $\mathfrak{g} \otimes \mathfrak{g}^{\vee}$ there exists a degree $m$ invariant polynomial $v$ on $\mathfrak{g}$ such that

$$
p_{\xi, m}(X)=u(\xi) \cdot v(X) \text {. }
$$

Because $p_{\xi, m}(-)$ is nontrivial for a generic $\xi$ we get that the polynomial $v$ is nonzero. Hence $p_{\xi, m}(X)$ is trivial if and only if $u(\xi)=0$.

Corollary 3.1. If $m$ in the above proposition is odd then there exists a coadjoint orbit $M_{\xi}$ for which the class $\mu_{2 m}$ is trivial in $H^{2 m}(B G)$.

\subsection{Coadjoint orbits of $\mathrm{SU}(n)$ again.}

Proposition 3.2. The $\mu_{3}$ class in $H^{6}(B \mathrm{SU}(n))$ is trivial for the adjoint orbit of the diagonal matrix $\operatorname{diag}\left[X_{1}, \ldots, X_{n}\right] \in \mathfrak{s u}(n)$ if and only if $\sum X_{i}^{3}=0$. In particular, the class $\mu_{3}$ is trivial for the grassmannian $G(m, 2 m)$ of $m$-planes in $\mathbb{C}^{2 m}$ and certain flag manifolds.

Proof. The algebra $S(\mathfrak{s u}(n))^{S U(n)}$ of invariant polynomials is generated by the polynomials of the form

$$
\mathfrak{s u}(n) \ni X \mapsto \sum X_{i}^{k}
$$

where $X_{i} \in \mathbb{C}$ are the eigenvalues of the matrix $X$. Hence any invariant polynomial of degree three is up to a constant equal to $\sum X_{i}^{3}$.

It follows from Proposition 3.1 that the orbit of a vector for which the above polynomial is trivial has vanishing class $\mu_{3}$. This include grassmannians $\mathrm{G}(m, 2 m)$ since it is (up to a scalar) the adjoint orbit of the diagonal matrix $X=\operatorname{diag}[i, \ldots, i,-i, \ldots,-i]$. Taking a generic zero of the above polynomial we obtain flag manifolds for which the class $\mu_{3}$ is trivial.

3.4. Coadjoint orbits of $\mathbf{S O}(2 n)$. Let $\mathrm{SO}(2 n) \rightarrow \mathrm{SO}(2 n+1) \rightarrow S^{2 n}$ be the bundle of the orthonormal frames with respect to the standard round metric. The associated bundle

$$
\mathrm{SO}(2 n) / U(n) \rightarrow E \rightarrow S^{2 n}
$$

admits a fibrewise symplectic form due to [9] (see also [4] for a more general statement). This symplectic form restricts to the symplectic form 
on the fibres and it represents the coupling class. Since it is nondegenerate the fibre integral of its top power is nonzero in $H^{2 n}\left(S^{2 n}\right)$. Thus the class $\mu_{n}(E)$ is a nonzero multiple of the Euler class of the tangent bundle of $S^{2 n}$.

It follows that the class $\mu_{n}$ is also nontrivial for an orbit in a neighbourhood of $\mathrm{SO}(2 n) / U(n)$. In particular, for every subgroup $H=\prod U\left(n_{i}\right)$, with $\sum n_{i}=n$, there is an $\mathrm{SO}(2 n)$-invariant form on $\mathrm{SO}(2 n) / H$ such that the class $\mu_{n}$ is nonzero.

Example 3.1. Let $M=\mathrm{SO}(8) / U(4)$. The above argument shows that the class $\mu_{4} \in H^{8}(B \operatorname{Ham}(M))$ is indecomposable. Moreover, the class $\mu_{2}$ is nontrivial, due to Corollary 2.2. Hence these classes are algebraically independent.

3.5. Coadjoint orbits of $\mathbf{S O}(4 n+2)$. Let $\mathfrak{t} \subset \mathfrak{s o}(4 n+2)$ be the Lie algebra of the maximal torus given by the skew symmetric matrices with $2 \times 2$-blocks on the diagonal. Hence an element $\xi \in \mathfrak{t}$ may be represented by an $(2 n+1)$-tuple $\left[t_{1}, \ldots, t_{2 n+1}\right]$ of real numbers.

We have $\operatorname{dim} \pi_{4 n+2}(B \mathrm{SO}(4 n+2)) \otimes \mathbb{Q}=1$ and the corresponding characteristic class is defined by the Pfaffian. When restricted to the Lie algebra $\mathfrak{t}$, the Pfaffian is, up to a constant, equal to the product of coordinates. This proves the following.

Proposition 3.3. Let $\xi=\left[t_{1}, t_{2}, \ldots, t_{2 n+1}\right] \in \mathfrak{t} \subset \mathfrak{s o}(4 n+2)$. The class $\mu_{2 n+1} \in H^{4 n+2}(B S O(4 n+2))$ is trivial for the coadjoint orbit $M_{\xi}$ if and only if $\prod t_{i}=0$.

\section{An application to lattices in semisimple groups}

Let $G$ be a semisimple Lie group, $K \subset G$ a maximal compact subgroup and $\Gamma \subset G$ an irreducible cocompact lattice trivially intersecting $K$. Let $M \subset G^{\mathrm{c}}$ be a maximal compact subgroup of the complexification of $G$. Let $H \subset K$ be the isotropy subgroup of $\xi \in \mathfrak{k}^{\vee}$. We have a Hamiltonian bundle

$$
K / H \rightarrow \Gamma \backslash G / H \rightarrow \Gamma \backslash G / K=B \Gamma
$$

classified by a map $\Gamma \backslash G / K \rightarrow B K$. It was observed by Okun [8] that this classifying map lifts to a map $\Gamma \backslash G / K \rightarrow M / K$ after passing to a sublattice of finite index if necessary. Let us call this lift the Okun map. The homomorphism $H^{*}(M / K) \rightarrow H^{*}(\Gamma)$ induced by the Okun map is called the Matsushima homomorphism and it is known to be injective. It is also surjective in degrees smaller than the rank of $G$. This result was proved by Matsushima in [6]. 
We obtain the following diagram of Hamiltonian fibrations in which the columns (but not the rows) are fibrations.

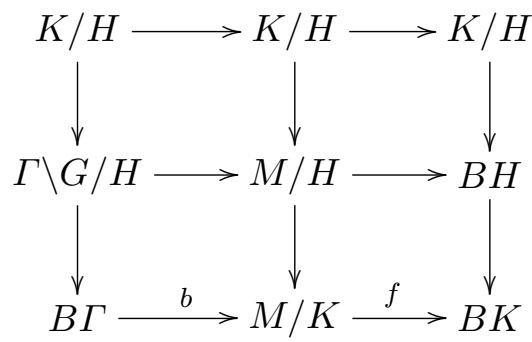

Theorem 4.1. Let $\xi \in \mathfrak{k}^{\vee}$ be a generic element such that its isotropy subgroup $H \subset K$ is a maximal torus. If $\pi_{2 k}(M / K) \otimes \mathbb{Q} \rightarrow \pi_{2 k}(B K) \otimes \mathbb{Q}$ is nontrivial then the characteristic class

$$
\mu_{k}(\Gamma \backslash G / H) \in H^{2 k}(B \Gamma)
$$

is nonzero.

Proof. Let $\sigma \in \pi_{2 k}(M / K) \otimes \mathbb{Q}$ be an element such that $f_{*}(\sigma) \neq 0$. According to Lemma 2.1 we have that the class $\mu_{k}(B H) \in H^{2 k}(B K)$ evaluates non trivially on $f_{*}(\sigma)$ hence the corresponding characteristic class $\mu_{k}(M / H)$ class is nonzero in $H^{2 k}(M / K)$. Since the Matsushima homomorphism is injective we obtain the statement.

Theorem 4.2. Let $K$ be a simple compact group different from $\mathrm{SO}(4 n)$. Suppose it is of maximal rank in $G$. Let $\xi \in \mathfrak{k}^{\vee}$ be a generic element. Denote its isotropy subgroup by $H \subset K$. Then the image of the Matsushima homomorphism $H^{*}(M / K) \rightarrow H^{*}(B \Gamma)$ is generated by the characteristic classes $\mu_{k}(\Gamma \backslash G / H)$.

Proof. It follows from Corollary 1.1 that the classes $\mu_{k}$ generate the cohomology ring $H^{*}(B K)$. Since $K$ and $M$ have equal rank the homomorphism $H^{*}(B K) \rightarrow H^{*}(M / K)$ is surjective hence the ring $H^{*}(M / K)$ is also generated by the classes $\mu_{k}$ and so is its image in $H^{*}(\Gamma)$.

\section{References}

[1] V. Guillemin, E. Lerman and S. Sternberg, Symplectic fibrations and multiplicity diagrams, Cambridge University Press, Cambridge, 1996.

[2] T. Januszkiewicz and J. Kędra, Characteristic classes of smooth fibrations, (math.SG/0209288).

[3] J. Kędra and D. McDuff, Homotopy properties of Hamiltonian group actions, Geom. Topol. 9 (2005), 121-162 (electronic).

[4] J. Kędra, A. Tralle and A. Woike, On nondegenerate coupling forms, J. Geometry and Physics, to appear, http://arxiv.org/abs/1004.3699, 2010.

[5] F. Lalonde and D. McDuff, Symplectic structures on fiber bundles, Topology 42(2) (2003), 309-347. 
[6] Y. Matsushima, On Betti numbers of compact, locally sysmmetric Riemannian manifolds. Osaka Math. J. 14 (1962), 1-20.

[7] M. Mimura and H. Toda, Topology of Lie groups. I, II, Translations of Mathematical Monographs, 91, American Mathematical Society, Providence, RI, 1991, translated from the 1978 Japanese edition by the authors.

[8] B. Okun, Nonzero degree tangential maps between dual symmetric spaces, Algebr. Geom. Topol. 1 (2001), 709-718 (electronic).

[9] A.G. Reznikov, Symplectic twistor spaces. Ann. Global Anal. Geom. 11(2) (1993), 109-118.

[10] A.G. Reznikov, Characteristic classes in symplectic topology, Selecta Math. (N.S.), 3(4) (1997), 601-642, Appendix D by Ludmil Katzarkov.

Mathematical Institute, University of WrocŁaW

Pl Grunwaldzki 2/4, 50-384 WrocŁaW, Poland

E-mail address: sgal@math.uni.wroc.pl

Mathematical Sciences, University of Aberdeen, Aberdeen AB243UE, Scotland, UK, and

Institute of Mathematics, University of SzcZecin

Wielkopolska 15, 70-451 Szczecin, Poland

E-mail address: kedra@abdn.ac.uk

Mathematical Sciences, University of Warmia and Mazury,

ŻoŁnierska 14A, 10-561 Olsztyn, Poland

E-mail address: tralle@matman.uwm.edu.pl

Received 05/28/2010, accepted 06/23/2010

S.G. is partially supported by the MNISW grant N N201 541738. J.K. would like to thank Ran Levi for useful conversations which led to the proof of Lemma 2.1. A.T. would like to thank IHES and the Max-Planck-Institut for hospitality during the work on this paper. 
